\title{
Obliegenheiten im Versicherungsvertragsrecht aus historisch-vergleichender Perspektive
}

\author{
Von Phillip Hellwege, Augsburg*
}

\begin{abstract}
Inhaltsübersicht
I. Einleitung. . . . . . . . . . . . . . . . . . . . . . . . 865

II. Das geltende Recht . . . . . . . . . . . . . . . . . . . . . . . . . 866

1. Deutsches Recht . . . . . . . . . . . . . . . . . . . . . . . . 866

2. Englisches Recht . . . . . . . . . . . . . . . . . . . . . . . . 868

3. Zwischenergebnis ................... . 871
\end{abstract}

* Abgekürzt werden zitiert: John Birds, Modern Insurance Law ${ }^{8}$ (2010); Charles John Bunyon, The Law of Fire Insurance (1867) (zitiert: Fire Insurance); ders., The Law of Fire Insurance $^{2}$ (1875) (zitiert: Fire Insurance ${ }^{2}$ ); ders., The Law of Fire Insurance ${ }^{3}$ (1885) (zitiert: Fire Insurance $^{3}$ ); Malcolm Clarke, The Law of Insurance Contracts ${ }^{6}$ (2009); Raoul Colinvaux (-Robert Merkin), Law of Insurance ${ }^{9}$ (2010); Phillip Hellwege, Allgemeine Geschäftsbedingungen, einseitig gestellte Vertragsbedingungen und die allgemeine Rechtsgeschäftslehre (2010); John Lowry/Philip Rawlings/Robert Merkin, Insurance Law (2011); Peter MacDonald Eggers, Insurance, in: Joseph Chitty, On Contracts ${ }^{30}$ II (2008) (zitiert: Chitty [-MacDonald Eggers]); Peter MacDonald Eggers/Simon Picken/Patrick Foss, Good Faith and Insurance Contracts ${ }^{3}$ (2010); Evan J. MacGillivray, On Insurance Law ${ }^{11}$, hrsg. von Nicholas Legh-Jones/John Birds/David Owen (2008); Andrew McGee, The Modern Law of Insurance 2 (2006); Oliver Meixner/René Steinbeck, Versicherungsvertragsrecht ${ }^{2}$ (2011); Münchener Kommentar zum Versicherungsvertragsgesetz, hrsg. von Theo Langheid/Manfred Wandt I (2010) (zitiert: Münch. Komm. VVG [-Bearb.]); James Biggs Porter, The Laws of Insurance (London 1884); Versicherungsvertragsgesetz ${ }^{2}$, hrsg. von Wilfried Rüffer/Dirk Halbach/Peter Schimikowski (2011) (zitiert: Rüffer/Halbach/Schimikowski [-Bearb.]); Peter Schimikowski, Versicherungsvertragsrecht ${ }^{4}$ (2009); Manfred Wandt, Versicherungsrecht ${ }^{5}(2010)$.

Weitere Abkürzungen: $\mathrm{AG}=$ Appellationsgericht; $\mathrm{AGH}=$ Appellationsgerichtshof; BuschA = Archiv für Theorie und Praxis des Allgemeinen Deutschen Handels- und Wechselrechts; $\mathrm{HAG}=$ Handelsappellationsgericht; OAG = Oberappellationsgericht; OTR = Obertribunal; PEICL = Principles of European Insurance Contract Law; PreußVersZ = PreuBische Versicherungs-Zeitschrift; RheinA = Archiv für das Civil- und Criminal-Recht der Königlich-Preußischen Rheinprovinzen; StriethorstA = Archiv für Rechtsfälle, die zur Entscheidung des königlichen Ober-Tribunals gelangt sind; Wochenblatt $=$ Wochenblatt für merkwürdige Rechtsfälle in actenmäßigen Darstellungen aus dem Gebiete der Justizpflege und Verwaltung zunächst für das Königreich Sachsen; ZVersR = Zeitschrift für Versicherungsrecht. 
III. Das 19. Jahrhundert . . . . . . . . . . . . . . . . . . . . . . 873

1. Englisches Recht . . . . . . . . . . . . . . . . . . 873

2. Deutsches Recht . . . . . . . . . . . . . . . . . . 877

a) Die Rechtslage bis 1865 . . . . . . . . . . . . . . . . . 877

b) Entwicklungen ab $1865 \ldots \ldots \ldots$. . . . . . . . . . . . . . 882

c) Zwischenergebnis . . . . . . . . . . . . . . 886

IV. Schlussbetrachtungen . . . . . . . . . . . . . . . 887

Summary: Condition Precedent in Insurance Contract Law in Historical and Comparative Perspective . . . . . . . . . . . . . . . . . . 891

\section{Einleitung}

Einen Versicherungsnehmer treffen bei Anbahnung und Durchführung eines Versicherungsvertrags sowie bei und nach Eintritt des Versicherungsfalles Verhaltensanforderungen: Vor Vertragsschluss muss er Informationen offenbaren, die der Versicherer zur Einschätzung des zu übernehmenden Risikos benötigt. Während der Laufzeit des Vertrags ist er etwa gehalten, risikoerhöhende Handlungen zu unterlassen. Und er hat den Versicherungsfall nach dessen Eintritt anzuzeigen und offenzulegen, was der Versicherer für dessen Regulierung benötigt. Diese Verhaltensanforderungen stehen im Zentrum der Dogmatik, ${ }^{1}$ und auch die vergleichende Forschung widmet sich ihnen immer wieder. ${ }^{2}$ Dabei offenbaren sich Unterschiede zwischen den europäischen Rechten, so mit Blick auf die Voraussetzungen, die erfüllt sein müssen, damit die an die Verletzung einer Verhaltensanforderung geknüpften Rechtsfolgen eintreten. Insbesondere wird die Frage unterschiedlich beantwortet, ob dafür ein Verschulden des Versicherungsnehmers erforderlich ist. Ein europäisches Versicherungsvertragsrecht muss versuchen, solche Unterschiede zu überwinden, und in der Tat sind erste Schritte in diese Richtung getan. ${ }^{3}$ Freilich bedarf es auch einer europäischen Versicherungsvertragsrechtswissenschaft, zum einen zur Vorbereitung eines europäischen Versicherungsvertragsrechts, zum anderen, um die für Europa gefundenen Regeln den Vertretern der nationalen Rechte zu vermitteln.

Reinhard Zimmermann steht wie kein anderer für einen historisch-vergleichenden Ansatz, um das Fundament einer europäischen Privatrechtswissenschaft zu legen und so den Weg hin zu einem europäischen Privatrecht zu ebnen. Als historische Parallele verweist er dafür auf den Kodifikations-

${ }^{1}$ Vgl. nur Reimer Schmidt, Die Obliegenheit (1953); Susanne Hähnchen, Obliegenheiten und Nebenpflichten (2010).

${ }^{2}$ Vgl. nur Giesela Rühl, Obliegenheiten im Versicherungsvertragsrecht (2004); Europäisches Versicherungsvertragsrecht, hrsg. von Jürgen Basedow/Till Fock I-III (2002-2003).

${ }^{3}$ Vgl. Principles of European Insurance Contract Law (PEICL), hrsg. von Jürgen Basedow/ John Birds et al. (2009); dazu Principles of European Insurance Contract Law, hrsg. von Helmut Heiss (2011). Vgl. auch Giesela Rühl, Die englischen warranties: ZEuP 14 (2006) 607-629. 
streit zwischen Thibaut und von Savigny zu Beginn des 19. Jahrhunderts. Damals habe sich die Ansicht durchgesetzt, dass zunächst die gemeinsamen historischen Wurzeln der in Deutschland geltenden Rechte herausgearbeitet werden müssten, bevor diese vereinheitlicht werden könnten. Heute gelte nichts anderes. Die gemeinsamen historischen Wurzeln der europäischen Privatrechte sieht er dabei im römisch-kanonischen ius commune. Eine europäische Privatrechtswissenschaft müsse diese Wurzeln herausarbeiten und nachverfolgen, wie und warum sich die einzelnen europäischen Privatrechte seit Beginn ihrer Nationalisierung im ausgehenden 18. Jahrhundert auseinanderentwickelt hätten. Der dabei erzielte Erkenntnisgewinn könne sodann die Debatte um ein europäisches Privatrecht befruchten. ${ }^{4}$

In der Versicherungsrechtswissenschaft wurde dieses historisch-vergleichende Programm bisher nicht rezipiert, sind doch auch die historischen Grundlagen andere. So spielte das römisch-kanonische ius commune als gemeineuropäisches Phänomen im Versicherungsrecht eine nur marginale Rolle. ${ }^{5}$ Der vorliegende Beitrag wird aufzeigen, dass das von Reinhard Zimmermann entwickelte Programm im Versicherungsrecht dennoch gewinnbringend genutzt werden kann. Dabei sollen die an den Versicherungsnehmer gestellten Verhaltensanforderungen in Deutschland und England im Zentrum stehen. Denn auch ${ }^{6}$ im Versicherungsvertragsrecht scheint die Überwindung der Unterschiede zwischen den kontinentaleuropäischen Rechten und dem englischen Recht große Probleme zu bereiten, und dabei scheinen insbesondere die an den Versicherungsnehmer gestellten Verhaltensanforderungen ein, wie Rühl es plastisch ausdrückt, Stolperstein auf dem Weg hin zu einem europäischen Versicherungsvertragsrecht zu sein. ${ }^{7}$

\section{Das geltende Recht}

\section{Deutsches Recht}

Das deutsche Recht fasst die Verhaltensanforderungen an den Versicherungsnehmer zusammen, bezeichnet sie als Obliegenheiten und versucht, sie einheitlichen Grundsätzen zu unterstellen. Freilich finden sich auch Diffe-

${ }^{4}$ Vgl. etwa Reinhard Zimmermann, Savignys Vermächtnis (1998); ders., Das römisch-kanonische ius commune als Grundlage europäischer Rechtseinheit: JZ 1992, 8-20 (8ff.).

${ }_{5}$ Zur Geschichte des Versicherungsrechts vgl. Peter Koch, Versicherungswesen, in: Handwörterbuch zur Deutschen Rechtsgeschichte V (1998) 815-826 (815ff.). Zur Geschichte des Versicherungsgedankens vgl. Albert Schug, Der Versicherungsgedanke und seine historischen Grundlagen (2010).

${ }^{6}$ Allgemein zum Vorurteil grundsätzlicher Verschiedenheit Reinhard Zimmermann, Römisches Recht und europäische Kultur: JZ 2007, 1-12 (1 ff.).

${ }^{7}$ Rühl (oben N. 3) 607 ff. Freilich meint Rühl, dass diese Unterschiede kein Stolperstein seien, siehe unten den Text zu N. 47. 
renzierungen - so zwischen vertraglichen und gesetzlichen Obliegenheiten. ${ }^{8}$

Grundnorm für vertraglich begründete Obliegenheiten ist $\$ 28$ VVG. Erfasst werden vor allem die Obliegenheiten, dass der Versicherungsnehmer gefahrerhöhende Handlungen zu unterlassen, Gefahrerhöhungen anzuzeigen und nach Eintritt des Versicherungsfalls die nötigen Informationen zu geben hat. ${ }^{9} \$ 28$ VVG sieht sodann zwei mögliche Rechtsfolgen bei einer Obliegenheitsverletzung vor: die Kündigung durch den Versicherer und die Befreiung von seiner Leistungspflicht. Verletzt der Versicherungsnehmer eine vertragliche Obliegenheit schuldlos oder leicht fahrlässig, so treten diese Rechtsfolgen nicht ein. Verletzt er eine Obliegenheit, die vor Eintritt des Versicherungsfalles zu erfüllen ist, vorsätzlich oder grob fahrlässig, so kann der Versicherer den Vertrag nach $\$ 28$ I VVG innerhalb eines Monats ab Kenntniserlangung von dieser Verletzung fristlos kündigen. ${ }^{10}$ Sieht der Vertrag vor, dass der Versicherer bei der Verletzung einer Obliegenheit von seiner Leistungspflicht befreit wird, so tritt diese Rechtsfolge nach $\$ 28$ II 1 VVG nur bei Vorsatz auf Seiten des Versicherungsnehmers ein. ${ }^{11}$ Hat dieser dagegen die vertragliche Obliegenheit grob fahrlässig verletzt, kommt es nach $\$ 28$ II 2 VVG zu einer bloßen Leistungskürzung. ${ }^{12}$ Freilich setzen Leistungsbefreiung und Leistungskürzung auch eine Ursächlichkeit zwischen der Obliegenheitsverletzung einerseits und dem Eintritt oder der Feststellung des Versicherungsfalles bzw. der Feststellung oder dem Umfang der Leistungspflicht des Versicherers andererseits voraus, $\$ 28$ III 1 VVG. Nur für den Fall der Arglist macht $\$ 28$ III 2 VVG von diesem Kausalitätserfordernis eine Ausnahme.

Für gesetzliche Obliegenheiten fehlt eine vergleichbare Grundnorm. \19 VVG etwa behandelt vorvertragliche Anzeigeobliegenheiten. Danach erfordert ein Rücktritt Vorsatz oder grobe Fahrlässigkeit, ${ }^{13}$ eine bloße Kündigung ist hingegen auch bei einfacher Fahrlässigkeit und fehlendem Verschulden möglich. ${ }^{14}$ Eine Kündigung wegen subjektiver Risikoerhöhung

${ }^{8}$ Wandt Rz. 564, 553.

9 Rüffer/Halbach/Schimikowski (-Joachim Felsch) \$28 Rz.3.

${ }_{10}$ Rüffer/Halbach/Schimikowski (-Felsch) \$28 Rz. 125; Erich R. Prölss/Anton Martin (-Jürgen Prölss), Versicherungsvertragsgesetz ${ }^{28}$ (2010) $\$ 28$ Rz. 100 (zitiert: Prölss/Martin [-Bearb.]); Meixner/Steinbeck \$6 Rz. 125; Münch. Komm. VVG (-Manfred Wandt) $\$ 28 \mathrm{Rz} .174 \mathrm{ff}$.

${ }_{11}$ Prölss/Martin (-J. Prölss) (vorige Note) $\$ 28$ Rz. 112; Hans-Peter Schwintowski/Christoph Brömmelmeyer (-Schwintowski), Praxiskommentar zum Versicherungsvertragsrecht ${ }^{2}$ (2011) $\mathbb{} \int 8$ Rz. 19 (zitiert: Schwintowski/Brömmelmeyer [-Bearb.]); Meixner/Steinbeck \6 Rz. 155.

12 Wandt Rz. 596 ff.; Münch. Komm. VVG (-Wandt) \$28 Rz. 230; Prölss/Martin (-J. Prölss) (oben N.10) $\$ 28$ Rz.112; Schwintowski/Brömmelmeyer (-Schwintowski) (vorige Note) $\$ 28$ Rz. 51.

${ }^{13}$ Wandt Rz. 620 ff.; Schimikowski Rz. 190; Meixner/Steinbeck $\$ 6$ Rz.92; Münch. Komm. VVG (-Theo Langheid) \$ 19 Rz. 128.

14 Rüffer/Halbach/Schimikowski (-Peter Schimikowski) $\$ 19$ Rz.38; Meixner/Steinbeck $\$ 6$ Rz. 98; Münch. Komm. VVG (-Langheid) \$19 Rz.137; Schimikowski Rz. 190. 
setzt nach $\$ 24$ I VVG einfache Fahrlässigkeit voraus, wobei mit Blick auf die Kündigungsfristen zwischen verschiedenen Verschuldensgraden differenziert wird. ${ }^{15}$ Eine Leistungsbefreiung erfordert Vorsatz, wohingegen es bei grober Fahrlässigkeit zu einer Kürzung des Leistungsanspruchs kommt, $\mathbb{} 26$ VVG. ${ }^{16}$ Zudem verlangen auch die gesetzlichen Obliegenheiten grundsätzlich Ursächlichkeit.

In der Gesamtschau versucht der Gesetzgeber, im Recht der Obliegenheiten die sich gegenüberstehenden Interessen angemessen zum Ausgleich zu bringen. ${ }^{17}$ Er tat dies bereits im VVG von 1908 durch Anerkennung des Verschuldensprinzips. Mit der Reform von 2008 hat er das Alles-odernichts-Prinzip überwunden und stuft nunmehr nach Verschuldensgraden ab. Zudem ist der Ansatz des deutschen Gesetzgebers, Obliegenheiten einheitlichen Grundsätzen zu unterstellen.

\section{Englisches Recht}

Auch das englische Recht stellt an den Versicherungsnehmer Verhaltensanforderungen. Bei Vertragsanbahnung trifft ihn eine gesetzliche duty of disclosure. ${ }^{18}$ Sie wird aus der Natur des Versicherungsvertrages als Vertrag uberrimae fidei, als Vertrag of utmost good faith, hergeleitet. ${ }^{19}$ Der Versicherungsnehmer muss danach alle vertragserheblichen Umstände anzeigen. Was vertragserheblich ist, bestimmt sich aus Sicht eines prudent insurer. ${ }^{20}$ Was die Parteien für erheblich halten, ist ohne Relevanz. ${ }^{21}$ Ohne Bedeutung ist auch, ob der Versicherungsnehmer die Erheblichkeit erkennen konnte. ${ }^{22}$

15 Meixner/Steinbeck $₫ 6$ Rz. 204f.; Münch. Komm. VVG (-Wolfram Wrabetz/Peter Reusch) $\$ 24$ Rz.3; Schimikowski Rz.205; Schwintowski/Brömmelmeyer (-Leander Loacker) (oben N. 11) $\$ 24$ Rz. 5.

${ }_{16}$ Meixner/Steinbeck \6 Rz.221f.; Schimikowski Rz.206; Rüffer/Halbach/Schimikowski (-Christoph Karczewski) \26 Rz. $1 \mathrm{ff}$.

17 Wandt Rz. 564, 597.

${ }^{18}$ Hierzu MacGillivray paras. 17-004ff.; Chitty (-MacDonald Eggers) paras. 41-031ff.; Birds paras. $7.3 \mathrm{ff}$; Colinvaux (-Merkin) paras. 6-02 ff.; McGee paras. $5.3 \mathrm{ff}$.

19 Vgl. nur Birds para. 7.3; McGee para. 5.3; Colinvaux (-Merkin) para. 6-001; Semin Park, The Duty of Disclosure in Insurance Contract Law (1996) para. 1. Zum Prinzip der uberrima fides aus historischer Sicht Nicole Schneider, Uberrima Fides, Treu und Glauben und vorvertragliche Aufklärungspflichten im englischen Recht (2004).

${ }^{20}$ Lowry/Rawlings/Merkin 89; MacGillivray para. 17-035; Chitty (-MacDonald Eggers) para. 41-031; McGee para. 5.4; Robert Merkin (-John Lowry), Insurance Law (2007) 39 (zitiert: Merkin [-Bearb.]); Lambert v. Co-operative Insurance Society Ltd. [1975] 2 Lloyd's Rep. 485. Beispiele vertragserheblicher Umstände finden sich bei McGee paras. $5.19 \mathrm{ff}$; Colinvaux (-Merkin) paras. 6-045ff.; Birds paras. $7.11 \mathrm{ff}$.

${ }^{21}$ Chitty (-MacDonald Eggers) para. 41-031; MacGillivray para. 17-039; Birds paras. 7.6, 7.8; Lambert v. Co-operative Insurance Society Ltd. (vorige Note) 485.

${ }^{22}$ Lowry/Rawlings/Merkin 89; Birds para. 7.6; Colinvaux (-Merkin) para. 6-022; Zeller v. British Caymanian Insurance Co. Ltd. (Cayman Islands) [2008] UKPC 4. 
Zudem können Umstände vertragserheblich sein, nach denen der Versicherer nicht gefragt hat. ${ }^{23}$ Freilich beschränkt sich die duty of disclosure auf Umstände, die dem Versicherungsnehmer bekannt sind ${ }^{24}$ bzw., nach umstrittener Ansicht, die er kennen muss. ${ }^{25}$ Umstände, die dem Versicherer bekannt sind oder die dieser kennen muss, braucht der Versicherungsnehmer nicht anzuzeigen. ${ }^{26}$ Auf ein Verschulden auf Seiten des Versicherungsnehmers kommt es nicht an. ${ }^{27}$ Die Nichtanzeige eines vertragserheblichen Umstandes muss freilich mitursächlich für den Vertragsschluss sein. ${ }^{28}$ Verletzt der Versicherungsnehmer auf diese Weise die duty of disclosure, kann der Versicherer den Vertrag mit Wirkung ex tunc anfechten..$^{29}$ Als Folge kann der Versicherer seine Leistung verweigern, muss aber die bereits erlangten Versicherungsprämien zurückzahlen, ${ }^{30}$ außer der Versicherungsnehmer hat arglistig gehandelt. ${ }^{31}$ Auch Versicherungssummen aus bereits regulierten Versicherungsfällen muss der Versicherungsnehmer zurückzahlen. ${ }^{32}$ In der Gesamtschau läuft der Versicherungsnehmer also Gefahr, seinen Versicherungsschutz ex tunc zu verlieren, wenn er einen Umstand verschweigt, der ihm nur bekannt sein musste und den er ohne Schuld, etwa weil der Versicherer nicht nach ihm gefragt hat, für nicht vertragserheblich gehalten hätte, wenn er von ihm positive Kenntnis gehabt hätte. Und der Versicherer kann nach Anfechtung die Regulierung eines Versicherungsfalles auch ablehnen, wenn der nicht angezeigte Umstand für diesen Versicherungsfall ohne jede Bedeutung ist.

Aus dem Prinzip des utmost good faith ergeben sich auch bei Vertragsdurchführung Verhaltensanforderungen. Die vorherrschende Meinung betont aber, dass diese in den verschiedenen Vertragsphasen von unterschiedlicher

23 MacGillivray para. 17-017; McGee para. 5.5; Chitty (-MacDonald Eggers) para. 41-031; Birds para. 7.7.2; Glicksman v. Lancashire \& General Assurance Co. Ltd. [1927] A. C. 139.

${ }^{24}$ MacGillivray paras. 17-009ff.; Birds para. 7.6.1.

25 Vgl. MacDonald Eggers/Picken/Foss paras. 7.92ff.; Chitty (-MacDonald Eggers) para. 41031; Economides v. Commercial Union Assurance Co. plc. [1997] 3 All E. R. 636.

26 Lowry/Rawlings/Merkin 115; Chitty (-MacDonald Eggers) para. 41-033; Birds para. 7.7; McGee paras. 5.33 ff.; Colinvaux (-Merkin) paras. 6-064ff.; Merkin (-Lowry) (oben N. 20) 52; London General Insurance Co. v. General Marine Underwriters' Association [1921] 1 K. B. 104.

27 MacGillivray para. 17-016; Birds para. 7.6.

28 MacGillivray para. 17-028; Birds para. 7.10; McGee para. 5.12; Colinvaux (-Merkin) paras. 6-027ff.; Pan Atlantic Insurance Co. Ltd. v. Pine Top Insurance Co. Ltd. [1994] 3 All E. R. 581; Assicurazioni Generali SpA v. Arab Insurance Group (BSC) [2002] EWCA Civ. 1642; Drake Insurance plc. v. Provident Insurance plc. [2003] EWCA Civ. 1834.

29 Lowry/Rawlings/Merkin 90; MacGillivray para. 17-008, paras. 17-029ff.; Colinvaux (-Merkin) para. 6-075; Chitty (-MacDonald Eggers) para. 41-039; Park (oben N. 19) para. 2.1.3.2; McGee para. 5.45; Black King Shipping Co. v. Massie (The Litsion Pride) [1985] 1 Lloyd's Rep. 437.

30 MacGillivray para. 17-030; Colinvaux (-Merkin) para. 6-075.

31 Lowry/Rawlings/Merkin 90; MacGillivray para. 16-005; Colinvaux (-Merkin) para. 6075 .

32 MacGillivray para. 17-030; Colinvaux (-Merkin) para. 6-075. 
Intensität seien, und ist zurückhaltend, weitere gesetzliche Verhaltensanforderungen zu formulieren. ${ }^{33}$ Während der Vertragsdurchführung sowie bei und nach Eintritt des Versicherungsfalles werden Verhaltensanforderungen daher vor allem aus vertraglichen Abreden, insbesondere warranties und conditions precedent, hergeleitet. Die Einordnung einer Verhaltensanforderung als warranty oder condition ist vertraglich disponibel, also eine Frage der Vertragsauslegung, ${ }^{34}$ wobei die Versicherungspraxis gewissen Mustern folgt.

Warranties haben regelmäßig zum Inhalt, dass der Versicherungsnehmer risikoerhöhende Handlungen nicht vornimmt und risikoerhöhende Umstände anzeigt. ${ }^{35}$ Werden sie verletzt, ist der Vertrag ipso iure und ex nunc aufgelöst. ${ }^{36}$ Auf ein Vertretenmüssen auf Seiten des Versicherungsnehmers kommt es nicht an. ${ }^{37}$ Und der Versicherer kann die Regulierung eines Versicherungsfalles auch ablehnen, wenn zwischen der Verletzung der warranty und dem Eintritt des Versicherungsfalles kein ursächlicher Zusammenhang besteht. ${ }^{38}$

Die Verletzung einer condition precedent führt zur bloßen Leistungsbefreiung auf Seiten des Versicherers, oder anders ausgedrückt, ihre Erfüllung ist Bedingung für das Entstehen des Anspruchs gegen den Versicherer. ${ }^{39}$ Typischerweise werden in ihnen Verhaltensanforderungen statuiert, die den Versicherungsnehmer bei und nach Eintritt des Versicherungsfalles treffen. ${ }^{40}$ So hat der Versicherungsnehmer den Versicherungsfall anzuzeigen und die für die Schadensregulierung notwendigen Auskünfte zu erteilen. Die Rechtsfolge der Verletzung einer condition precedent hängt wiederum nicht von einem Verschulden ab. ${ }^{41}$ Selbst die Unmöglichkeit, sich konform mit der condition precedent $\mathrm{zu}$ verhalten, ist unerheblich, außer diese Unmöglichkeit lag bereits bei Vertragsschluss vor. ${ }^{42}$ Bisher nicht entschieden ist die Frage,

${ }^{33}$ Vgl. Colinvaux (-Merkin) paras. 6-107ff.; McGee paras. $13.1 \mathrm{ff}$; Birds para. 7.15; Manifest Shipping Co. Ltd. v. Uni-Polaris Shipping Co. Ltd. (The Star Sea) [2001] UKHL 1; K/S MercScandia XXXXIIv. Certain Lloyd's Underwriters (The Mercandian Continent) [2001] EWCA Civ. 1275; Agapitos v. Agnew (The Aegeon) [2002] EWCA Civ. 247.

${ }^{34}$ Hierzu vgl. Chitty (-MacDonald Eggers) paras. 41-059f.; Clarke para. 20-2; HIH Casualty E General Insurance Ltd. v. New Hampshire Insurance Co. [2001] EWCA Civ. 735.

${ }_{35} \mathrm{Vgl}$. die Beispiele bei Birds para. 9.3.2.

${ }^{36}$ MacGillivray paras. 10-005, 10-022; Chitty (-MacDonald Eggers) para. 41-061; Birds para. 9.2; Colinvaux (-Merkin) para. 7-039; Bank of Nova Scotia v. Hellenic Mutual War Risks Association (Bermuda) Ltd. (The Good Luck) [1991] 2 W. L. R. 1279.

${ }^{37}$ MacGillivray paras. 10-022, 10-044ff.; Chitty (-MacDonald Eggers) para. 41-061; Birds para. 9.1; International Management Group (UK) Ltd. v. Simmonds [2003] EWHC 177.

${ }_{38}$ Clarke para. 20-3; Chitty (-MacDonald Eggers) para. 41-061; Birds paras. 6.4, 9.2; Merkin (-Alison Green) (oben N. 20) 87; Yorkshire Insurance Co. Ltd. v. Campbell [1916] UKPC 87.

39 MacDonald Eggers/Picken/Foss para. 8.1; Chitty (-MacDonald Eggers) para. 41-060.

${ }^{40}$ Birds paras. 9.9, 14.5ff.; MacGillivray paras. 10-010f.; Colinvaux (-Merkin) para. 7-004.

${ }^{41}$ Birds para. 14.6.1; Clarke para. 26-2E1; Chitty (-MacDonald Eggers) para. 41-071; Walker v. Pennine Insurance Co. Ltd. [1979] 2 Lloyd's Rep. 139.

${ }^{42}$ Clarke paras. 20-6B2, 26-2E1. 
ob der Versicherer die Regulierung eines Schadens wegen Nichteinhaltung einer condition precedent verweigern kann, weil der Versicherungsnehmer selbst den Versicherungsfall nicht rechtzeitig angezeigt oder notwendige Auskünfte nicht rechtzeitig erteilt hat, wenn der Versicherer ohne Zutun des Versicherungsnehmers die entsprechenden Informationen erhalten hat. $^{43}$

Ist es für das Eintreten der Rechtsfolge einer Verletzung einer duty of disclosure, einer warranty und einer condition precedent also jeweils unerheblich, ob der Versicherungsnehmer die Verhaltensanforderung schuldlos verletzt hat, so wird die Berufung auf ihre Verletzung anders begrenzt, etwa im Wege einer restriktiven Auslegung der obliegenheitsbegründenden Vertragsklauseln. ${ }^{44}$ Zudem kann der Versicherer auf die Einhaltung der Verhaltensanforderung verzichten (waiver), oder er kann die Berufung auf die Verletzung verwirken (estoppel), wobei das Verhältnis zwischen beiden Rechtsinstituten unklar ist. ${ }^{45}$ Schließlich wird diskutiert, ob sich aus der Pflicht zur besonderen Treue, der auch der Versicherer ausgesetzt ist, nicht Einschränkungen des Anfechtungsrechts bei Verstoß gegen die duty of disclosure herleiten lassen. ${ }^{46}$

\section{Zwischenergebnis}

Ein wichtiger Unterschied zwischen deutschem und englischem Recht ist nach allem die Bedeutung des Verschuldens des Versicherungsnehmers bei Verletzung einer Verhaltensanforderung. Im englischen Recht treten die an eine solche Verletzung geknüpften Rechtsfolgen auch dann ein, wenn dem Versicherungsnehmer kein Verschulden zur Last fällt. Das englische Recht bringt damit die sich gegenüberstehenden Interessen zu einem Ausgleich, der im Vergleich zum deutschen Recht für den Versicherungsnehmer ungünstiger ausfällt. Betrachten wir vor diesem Hintergrund die Principles of European Insurance Contract Law (PEICL), so stellen wir fest, dass sie insoweit nicht dem englischen Modell folgen: So führt etwa eine Verletzung der Verhaltensanforderung, dass der Versicherungsnehmer nach Eintritt des Versicherungsfalles bei dessen Aufklärung in angemessener Weise mitwirken soll, nur im Fall der Schädigungsabsicht und bei Rücksichtslosigkeit zu

${ }^{43}$ Birds para. 14.7.1; Clarke para. 26-2E1.

${ }^{44}$ Lowry/Rawlings/Merkin 134 ff.; Birds paras. 6.5, 7.7.2; Chitty (-MacDonald Eggers) para. 41-064; Drake Insurance plc. v. Provident Insurance plc. (oben N.28) 1834.

45 Birds para. 14.11; Clarke para. 20-7; Kosmar Villa Holidays plc. v. Trustees of Syndicate 1243 [2008] EWCA Civ. 147.

${ }^{46}$ Birds para. 8.2; Strive Shipping Corp. v. Hellenic Mutual War Risks Association [2002] EWHC 203; Brotherton v. Aseguradora Colseguros S. A. [2003] EWCA Civ. 705; Drake Insurance plc. v. Provident Insurance plc. (oben N.28) 1834. 
einem vollständigen Ausschluss des Anspruchs auf die Versicherungssumme und im Übrigen nur zu einer Leistungskürzung: Art. 6:102 PEICL.

Wird der Ansatz der PEICL ein Stolperstein auf dem Weg hin zu einem europäischen Versicherungsvertragsrecht sein? Giesela Rühl verweist darauf, englisches und deutsches Recht seien sich ähnlicher, als dies auf den ersten Blick scheine. ${ }^{47}$ Dies liege etwa an den Selbstverpflichtungen der Versicherer und der Regulierung durch die Financial Service Authority. Beides überlagere das Versicherungsvertragsrecht. Als Folge fielen die Unterschiede in der Praxis geringer aus, als ein Blick in das einschlägige Fallrecht vermuten lasse. Rühl kommt daher zu dem Ergebnis, dass die Unterschiede zwischen englischem Recht und deutschem Recht kein »Hindernis für die Harmonisierung des Versicherungsvertragsrechts in Europa [...] darstellen «. ${ }^{48}$

Jedoch darf nicht übersehen werden, dass sich der englische Gesetzgeber trotz Kritik an der Strenge gegenüber dem Versicherungsnehmer nach bestehender Rechtslage ${ }^{49}$ und trotz entsprechender Reformbestrebungen seit den 1950er Jahren ${ }^{50}$ bisher nicht zu einer umfassenden Reform durchringen konnte. Die Versicherungsindustrie konnte solche Reformen gerade durch die erwähnten Selbstverpflichtungen abwenden. Auch der im Mai 2011 vorgelegte Consumer Insurance (Disclosure and Representations) Bill wird keine umfassende Abhilfe bringen. Der Entwurf betrifft vor allem die sogenannte duty of disclosure und soll bewirken, dass nur noch solche Umstände vertragserheblich und zu offenbaren sind, nach denen der Versicherer fragt. ${ }^{51}$ Zudem sieht der Entwurf ein Verschuldenserfordernis vor, und würde schließlich auch auf Rechtsfolgenseite mehr Flexibilität bringen. ${ }^{52}$ Die Versicherungsindustrie trägt diese Reform mit. Eine Reform des Rechts der warranties und der conditions precedent in Versicherungsverträgen ist in dem Gesetzesentwurf dagegen nicht vorgesehen und scheint auch unwahrscheinlich zu sein. $.^{53} \mathrm{Da}-$ her ist es auch wenig wahrscheinlich, dass englische Juristen insoweit einer Reform aus Europa positiv gegenüberstehen werden.

47 Rühl (oben N. 3) $607 \mathrm{ff}$.

48 Rühl (oben N. 3) 629.

49 Vgl. etwa Birds paras. 7.17, 9.13; McGee para. 5.48; Chitty (-MacDonald Eggers) paras. 41042, 41-063 ff.; MacDonald Eggers/Picken/Foss paras. $5.01 \mathrm{ff}$.

50 Zuletzt Law Commission/Scottish Law Commission, Consumer Insurance Law: Pre-Contract Disclosure and Misrepresentation (2009) mit Verweis auf ältere Arbeiten beider Kommissionen.

${ }^{51}$ Lowry/Rawlings/Merkin $144 \mathrm{ff}$.

52 Lowry/Rawlings/Merkin $146 \mathrm{f}$.

${ }^{53}$ Lowry/Rawlings/Merkin 249. Auch in Law Commission/Scottish Law Commission, Insurance Contract Law: Post Contract Duties and other Issues (2011) wird das Thema nicht behandelt. 


\section{Das 19. Jahrhundert}

Kann die historische Rechtsvergleichung helfen, diese Skepsis zu überwinden? Nachfolgend wird nicht die Entwicklung aller Verhaltensanforderungen historisch-vergleichend nachverfolgt, ist doch auch der einheitliche Ansatz des deutschen Rechts nicht selbstverständlich. Die hier einheitlich als Obliegenheiten qualifizierten Anforderungen sind in England mal duty, mal warranty und mal condition. Ein historischer Vergleich müsste daher auch differenzieren. Das rechtfertigt eine Beschränkung. Im Weiteren werden nur solche Verhaltensanforderungen betrachtet, die nach Eintritt des Versicherungsfalles auftreten und die das englische Recht als condition precedent versteht. Diese Auswahl basiert auf der Beobachtung, dass die englischen Reformbestrebungen das englische und deutsche Recht der vorvertraglichen Informationsanforderungen ohnehin annähern werden. Aus Gründen der Quellenlage werden Feuerversicherungen im Zentrum stehen. Der Darstellungszeitraum, das ausgehende 18. und das 19. Jahrhundert in England und die zweite Hälfte des 19. Jahrhunderts in Deutschland, ist Ergebnis einer zunächst breiter angelegten Quellenauswertung.

\section{Englisches Recht}

Ein früher Fall wurde im Jahr 1796 entschieden: Worsley v. Wood. ${ }^{54}$ Die Bedingungen der Phoenix Company sahen vor, dass

“[...] all persons assured sustaining any loss by fire [...] procure a certificate under the hands of the ministers and churchwardens and of some reputable householders of the parish not concerned in the loss, importing that they were acquainted with the character and circumstances of the person insured, and knew or believed that he by misfortune and without any kind of fraud or evil practice had sustained by such fire the loss and damage therein mentioned; [...]."

Der Versicherungsnehmer hatte nach dieser üblichen Klausel ${ }^{55}$ ein Leumundszeugnis beizubringen. Doch weigerten sich Pfarrer und Gemeindevorsteher, ein solches auszustellen. Der Versicherungsnehmer meinte nun, es komme auf die Einhaltung dieser Verhaltungsanforderung nicht an, wenn diese Weigerung ohne Grund oder fehlerhaft erfolgt sei. Es könne nur verlangt werden, dass er sich um das verlangte Zeugnis redlich bemühe. Lord

54 Worsley v. Wood (1796) 6 T.R. 710. Vgl. auch Oldman v. Bewicke (1785) 2 H.Bl. 578; Routledge v. Burrell (1789) 1 H.B1. 254.

55 Zur Verbreitung dieser Klausel Samuel Marshall, A Treatise on the Law of Insurance II (1802) (zitiert: Treatise) 704; ders., A Treatise on the Law of Insurance ${ }^{3}$ II (1823) 807 (zitiert: Treatise ${ }^{3}$ ); Charles Ellis, The Law of Fire and Life Insurance and Annuities (1832) 61 (zitiert: Law of Fire); ders., The Law of Fire and Life Insurance and Annuities ${ }^{2}$ (1846) 64f. (zitiert: Law of Fire $^{2}$ ). 
Kenyon Ch. J. stellte klar, dass die Versicherer mit solchen Klauseln schützenswerte Interessen verfolgten, nämlich einem Versicherungsbetrug vorzubeugen. Es handele sich um eine condition precedent, die exakt erfüllt werden müsse. Der Versicherungsnehmer könne daher nicht ein anderes Leumundszeugnis vorlegen. Denn er habe bei Vertragsschluss gewusst, worauf er sich einlasse. Ashhurst J. ergänzte: ${ }^{56}$

"The question is whether the certificate required be or be not a condition precedent; if it be, the plaintiffs below cannot maintain their action. It is perfectly immaterial whether they have entered into an improvident contract; if they choose to take the burden upon themselves, they cannot call on the insurance office until they have complied with the condition. I think it is a condition precedent [...]."

Die Literatur verwies ebenfalls auf die Gefahr eines Versicherungsbetruges, glaubte diese Gefahr durch ein Leumundszeugnis abgemildert und hielt die Klausel daher für angemessen. ${ }^{57}$ Als condition precedent sei die Klausel auch strikt einzuhalten. Insbesondere könne die stipulierte Bedingung nicht durch eine gleichwertige Bedingung ersetzt werden, die den gleichen Zweck erfülle, und es sei unbeachtlich, wenn etwa der Gemeindepfarrer das Zeugnis zu Unrecht verweigere. Doch gab es auch kritische Stimmen. Dowdeswell schrieb 1846: ${ }^{58}$

"These requisitions form a condition precedent to any liability on the part of the insurers, and no action can be maintained until they have been complied with, even though the assured may be prevented from fulfilling them by the vexatious or capricious refusal of a person over whom he can exercise no control, to grant the certificate. The insertion, therefore, of clauses of a similar nature to those first alluded to is obviously objectionable, and although they were common in the older policies they have now most properly fallen into comparative desuetude."

Die Bedingung, dass ein Leumundszeugnis beizubringen sei, wurde also unüblich. ${ }^{59}$ Die weiterhin üblichen Verhaltensanforderungen wurden nach wie vor als condition precedent eingeordnet, so etwa in Mason v. Harvey aus dem Jahr 1853. ${ }^{60}$ Der Versicherungsnehmer hatte es unterlassen, so wie es die Versicherungsbedingungen der Norwich Union Fire Insurance Society vorsahen, innerhalb von drei Monaten eine Schadensaufstellung einzureichen. Der Versicherungsnehmer war der Ansicht, es handele sich nicht um

56 Worsley v. Wood (oben N. 54) 720.

57 Marshall, Treatise 705 f.; ders., Treatise 307 ff.; Ellis, Law of Fire $61 \mathrm{ff.;} \mathrm{ders.,} \mathrm{Law} \mathrm{of} \mathrm{Fire}{ }^{2}$ 65 ff. (alle oben N. 55); George Beaumont, The Law of Fire and Life Insurance (1833) 56, 99; Porter 183; David Hughes, A Treatise on the Law relating to Insurance (1828) $510 \mathrm{f}$.

58 George Morley Dowdeswell, The Law of Life and Fire Insurance (1846) $110 \mathrm{ff}$.

59 So auch der Hinweis bei Bunyon, Fire Insurance 90.

${ }^{60}$ Mason v. Harvey (1853) 8 Ex. 819. 
eine condition precedent, sondern um eine vertragliche Nebenpflicht. Pollock C.B. folgte dieser Auslegung nicht: ${ }^{61}$

"By the contract of the parties, the delivery of the particulars of loss is made a condition precedent to the right of the assured to recover."

In Roper v. Lendon, einer Entscheidung aus dem Jahr 1859, ging es schließlich um eine Klausel, diesmal der Kent Fire Insurance Company, wonach der Versicherungsfall unverzüglich anzuzeigen und innerhalb von fünfzehn Tagen eine Schadensübersicht einzureichen war. ${ }^{62}$ Die fünfzehn Tage ließ der Versicherungsnehmer verstreichen. Wiederum ging das Gericht davon aus, es handele sich um eine condition precedent. Werde die Schadensübersicht nicht innerhalb der stipulierten Frist eingereicht, entstehe der Anspruch des Versicherungsnehmers nicht. Die Literatur stand mit diesem Verständnis im Einklang: ${ }^{63}$ Sie hielt diese Klausel für angemessen, sei es doch dem Versicherer nur möglich, den Versicherungsfall zu überprüfen, wenn er schnell von ihm Kenntnis erlange; wiederum überwog die Angst vor einem Versicherungsbetrug auf Seiten des Versicherungsnehmers.

Bereits diese drei Urteile legen die Vermutung nahe, dass sich das englische Recht, was die Einordnung der uns interessierenden Klauseln als condition precedent angeht, seit dem ausgehenden 18. Jahrhundert nicht fortentwickelt hat. Zudem können wir erkennen, dass sich die Sorge vor einem Versicherungsbetrug wie ein roter Faden durch die Begründungen hindurchzieht. ${ }^{64}$ Freilich offenbart sich seit der zweiten Hälfte des 19. Jahrhunderts auch die Tendenz in der Rechtsprechung, Unbilligkeiten abzumildern. Die in Mason v. Harvey streitentscheidende Klausel lautete:

"Whenever any fire shall happen, the party insured shall [...] within three calendar months deliver [...] accounts exhibiting the full particulars and amount of the loss sustained $[\ldots] . "$

Der Kläger hatte sich darauf berufen, dass es unbillig sei, die Klausel als Bedingung einzuordnen, weil jeder noch so unbedeutende Fehler in der Schadensaufstellung dann dazu führen müsse, dass ein Anspruch nicht bestehe. Pollock C. B. entgegnete: ${ }^{65}$

"It has been argued that such a construction would be most unjust, since the plaintiff might be prevented from recovering at all by the accidental omission of some article. But the condition is not to be construed with such strictness. Its

${ }^{61}$ Mason v. Harvey (vorige Note) 821.

${ }^{62}$ Roper v. Lendon (1859) 1 El. \& El. 825.

${ }^{63}$ Bunyon, Fire Insurance 90; ders., Fire Insurance ${ }^{2} 102$; ders., Fire Insurance ${ }^{3} 135$; Porter $158 \mathrm{ff} ., 178 \mathrm{ff} ., 181$; Charles Francis Morrell, A Popular Statement of the Law of Insurance (1886) $43 \mathrm{f}$.

64 Porter 181.

${ }^{65}$ Mason v. Harvey (oben N. 60) 821. 
meaning is, that the assured will, within a convenient time after the loss, produce to the company something which will enable them to form a judgment as to whether or no he has sustained a loss. Such a condition is, in substance, most reasonable [...]."

Auffällig ist, dass Pollock C.B. die Klausel als angemessen billigt. Das kann man so deuten, dass sich die Zeitgenossen über die Wirksamkeit solcher Klauseln Gedanken machten und nicht Klauseln mit jedem Inhalt anerkannt hätten. Auch Porter deutet dies in seinem Lehrbuch zum Versicherungsrecht von 1886 an. ${ }^{66}$

Für die Tendenz der Gerichte, solche Klauseln seit der zweiten Hälfte des 19. Jahrhunderts einschränkend auszulegen, finden sich zudem weitere Beispiele: Die Bedingung, dass der Schaden zur Zufriedenheit des Versicherers nachgewiesen werden müsse, bedeute nicht, dass der Versicherer aus nicht nachvollziehbaren Gründen Nachweise zurückweisen dürfe. Es genüge, wenn sich ein vernünftiger Versicherer mit dem vorgelegten Nachweis zufriedengegeben hätte. ${ }^{67}$ In dem Fall Braunstein v. Accidental Death Insurance Co., einem Fall aus dem Unfallversicherungsrecht, begründete Wightman J. dieses Ergebnis wie folgt: ${ }^{68}$

"Now it is said that, by virtue of this clause, the directors have the power, if they please, wholly to withhold payment by capriciously [...] and without any reasonable ground whatsoever, requiring further evidence perfectly immaterial to the matter in dispute. The question is, whether, giving a reasonable construction to the intention of the parties when the clause was introduced by reference into the policy, it can be understood that the assured did agree that, upon any ground whatsoever, capriciously or otherwise, which the directors [...] might think fit to urge, their decision should be binding? The clause must receive a reasonable construction, and the parties must be taken to have had in view any further evidence which the directors might reasonably require; such a construction would fulfil all the terms of that clause."

Wightman J. führte weiter aus, dass in die Klausel, nach welcher der Versicherer weitere Beweise verlangen konnte, das Wort "reasonable« als implied hineingelesen werden müsse. Es ging bei der Auslegung also nicht darum, was der Versicherer nach dem Wortlaut der Klausel wirklich wollte, sondern darum, was die Parteien in angemessener Weise intendiert haben mussten. ${ }^{69}$

Konnte die Schadenshöhe nur durch Vorlage von Geschäftsbüchern nachgewiesen werden, so sollte es sich zwar um eine condition precedent handeln,

66 Porter 179.

67 Dowdeswell (oben N. 58) 110 ff.; Bunyon, Fire Insurance 91; James Biggs Porter, The Laws of Insurance ${ }^{3}$ (1898) 215.

68 Braunstein v. Accidental Death Insurance Co. (1861) 1 B. \& S. $794 \mathrm{f}$.

69 Braunstein v. Accidental Death Insurance Co. (vorige Note) 797 f. per Crompton J. 
aber englische Gerichte begnügten sich seit der zweiten Hälfte des 19. Jahrhunderts mit einem alternativen Schadensnachweis, wenn die Geschäftsbücher dem Brand ebenfalls zum Opfer gefallen waren. ${ }^{70}$ Freilich sahen englische Juristen eine Missbrauchsgefahr: ${ }^{71}$

" $[\ldots]$ in all conditions the exhibition of the books of account of trading firms is required, and if they are burnt or lost the next best proof, such as invoices or extracts from the books of correspondents, must be supplied. The loss of the books is generally considered a cause of suspicion by the assessors of fire losses."

Hier scheint wiederum die Angst vor einem Versicherungsbetrug durch. Zudem wies die englische Literatur darauf hin, dass die Rechtsprechung im Fall eines Auslegungsspielraums die Versicherungsbedingungen in angemessener Weise auslege. ${ }^{72}$ Und schließlich bildete sich die Ansicht heraus, der Versicherer könne auf die Erfüllung der Verhaltensanforderungen auch stillschweigend verzichten. ${ }^{73}$

\section{Deutsches Recht}

Wenden wir uns der Rechtsprechung in Deutschland zu, so offenbart sich, dass sie ebenso wie die englische davon ausging, der Anspruch des Versicherungsnehmers auf Zahlung der Versicherungssumme sei bedingt, und die Bedingung sei die Einhaltung der an den Versicherungsnehmer gestellten Verhaltensanforderungen. Doch anders als die englischen Gerichte standen deutsche Gerichte dieser dogmatischen Einordnung der Verhaltensanforderungen als Bedingung von Anfang an kritisch gegenüber, und im deutschen Recht vollzog sich zwischen 1865 und 1870 schlagartig ein entscheidender Wandel.

a) Die Rechtslage bis 1865

Ein erstes Urteil stammt aus dem Jahre 1847. Es handelt sich um ein Verfahren zwischen einem Stüttgen und der Kölnischen FeuerversicherungsGesellschaft, das vom Appellationsgerichtshof zu Köln entschieden wurde. ${ }^{74}$ Stüttgen und ein Eickenberg eröffneten 1844 eine Gastwirtschaft, für die sie bei der Beklagten am 23.10. 1844 eine Feuerversicherung abschlossen. Bereits am 24.11. 1844 brannte die Gastwirtschaft vollständig nieder. Die An-

70 Porter 188.

71 Bunyon, Fire Insurance ${ }^{3} 136$.

72 Bunyon, Fire Insurance ${ }^{3} 136$.

73 Bunyon, Fire Insurance ${ }^{3} 137$.

74 AGH Köln 28.6.1847, RheinA 42/1 (1847) 92. Vgl. auch AGH Köln 11.2.1863, RheinA 57/1 (1862) 114. 
zeige des Brandes und des Schadens erfolgten umgehend. Freilich war die begehrte Versicherungssumme zu hoch, weil auch gerettetes Mobiliar als zerstört gemeldet worden war. Eickenberg wurde deshalb in einem Strafverfahren verurteilt, vermutlich wegen Betruges, Stüttgen hingegen von entsprechenden Vorwürfen freigesprochen. Nach Abschluss des Strafverfahrens forderte Stüttgen die Versicherung am 1.10.1845 zur Regulierung des Schadens auf. Als diese ablehnte, erhob er am 27.12.1845 Klage auf Ersatz seines eigenen Schadens, also unter Ausschluss des Schadens des Eickenberg, vor dem Landgericht Düsseldorf. Die Beklagte berief sich auf $\mathbb{\$} 19$ ihrer Policebedingungen:

"Jeder nicht innerhalb 6 Monaten festgestellte oder nicht vor den Richter gebrachte Entschädigungsanspruch ist erloschen."

Diese Frist hatte der Kläger überschritten. Doch berief er sich darauf, dass er seine Klage nicht habe erheben können, solange das Strafverfahren nicht abgeschlossen gewesen sei. Im »Archiv für das Civil- und Criminal-Recht» wird sein Vortrag wie folgt zusammengefasst: ${ }^{75}$

»Der Kläger bestritt, daß der $\$$. 19 Platz greife; er habe sofort nach dem Brande den Schadensersatz gefordert, während der Untersuchung habe er nach Art. 3 der Crim. P. O. keine Civilklage erheben können, die Strafbestimmung des $\$$. 19 setze voraus, daß der Versicherte durch sein Verschulden die Frist nicht inne gehalten und dadurch auf den Entschädigungsanspruch stillschweigend verzichtet habe."

Das Landgericht Düsseldorf folgte der Argumentation des Klägers und befand, dass "die Strafclausel den Nachweis einer culpa oder eine böswilligen Absicht erfordere « ${ }^{76}$ Doch der Appellationsgerichtshof zu Köln gab der Berufung der Beklagten statt und wies die Klage ab. Er stellte fest, dass es auf ein Verschulden auf Seiten des Versicherungsnehmers nicht ankomme, und führte aus: ${ }^{77}$

„Daß es zu der Begründung der Anwendbarkeit dieser vertragsmäßigen Uebereinkunft der Parteien, einer vorherigen Versetzung des Appellaten in Verzug keineswegs bedurfte, da es sich hier nicht, wie der letztere behauptet, von der Vollziehung einer Pönal-Clausel im Sinne des Art. 1226 des B. G. B. ${ }^{[78]}$, sondern von der Erfüllung einer verabredeten Bedingung handelt, ohne deren Vorhandensein der Appellat einen Anspruch auf Entschädigung überhaupt nicht geltend machen konnte."

Ebenso wie die Versicherer in England berief sich die Kölnische Feuerversicherungs-Gesellschaft darauf, dass es sich bei der einzuhaltenden Frist

75 AGH Köln 28.6. 1847 (vorige Note) 93.

${ }^{76}$ LG Düsseldorf [ohne Datum], RheinA 42/1 (1847) 92 (93).

77 AGH Köln 28. 6. 1847 (oben N. 74) 95. Hervorhebung vom Verfasser.

78 Vgl. Art. 1226 Code civil. 
um eine Bedingung, oder, wie der englische Jurist sagen würde, eine condition precedent, handelt, und der Appellationsgerichtshof folgte dieser dogmatischen Einordnung der streitentscheidenden Klausel. Auf ein Verschulden auf Seiten des Versicherungsnehmers kam es als Folge nicht an. Doch deutet das erstinstanzliche Urteil bereits auf ein Unbehagen darüber hin, dass selbst eine unverschuldete Nichterfüllung der Bedingung dem Anspruch entgegenstehen sollte.

Die Klausel, dass der Versicherungsnehmer seine Klage innerhalb von sechs Monaten nach dem Schadensfall erheben muss, beschäftigte auch zahlreiche andere Gerichte, die noch eine weitere mögliche dogmatische Einordnung diskutierten: Wie war es, wenn der Versicherungsnehmer die Sechsmonatsfrist nur deshalb nicht einhielt, weil er in umfangreichen, aber am Ende nicht zielführenden Verhandlungen mit der Versicherung stand, die länger als sechs Monate andauerten? Erstinstanzliche Richter diskutierten die Möglichkeit, die Sechsmonatsfrist als Abrede über die Verjährung einzuordnen. Dies wäre für den Versicherungsnehmer aus gleich mehreren Gründen vorteilhaft gewesen. So waren Verjährungsabreden in Preußen nach ALR I 9 \$S 565-567 besonderen Wirksamkeitsvoraussetzungen ausgesetzt, die in Versicherungsbedingungen in der Regel nicht beachtet wurden. Doch blieb die vorherrschende Ansicht dabei, dass es sich um Bedingungen handelt. ${ }^{79}$

Die Einordnung als vertragliche Abrede über die Verjährung wäre noch aus einem anderen Grund für den Versicherungsnehmer von Vorteil gewesen. So enthielt das Allgemeine Landrecht für die Preußischen Staaten im Abschnitt zum Versicherungsvertrag besondere Vorschriften zur Verjährung. ALR II 8 \$2355 lautete:

"Sind über die Vergütung Unterhandlungen gepflogen worden: so wird die darauf verwendete Zeit, bis zu dem Zeitpunkte, da selbige wegen der Weigerung des Versicherers abgebrochen worden, in die Verjährungszeit nicht mit eingerechnet."

So hatte das Preußische Obertribunal Berlin im Jahre 1851 über einen Fall zu entscheiden, in dem nicht die Sechsmonatsfrist, sondern eine Jahresfrist versäumt worden war. ${ }^{80} \$ 18$ der Policebedingungen der Magdeburger Feuerversicherungs-Gesellschaft lautete:

"Alle nicht innerhalb Jahresfrist nach dem Brande entweder festgestellten oder vor Schiedsrichter gebrachten Ansprüche auf Entschädigung sind erloschen."

${ }^{79}$ OTR Berlin 5.6.1860, StriethorstA 38 (1861) 34; 7.6.1866, StriethorstA 64 (1867) 138; 11. 10. 1866, PreußVersZ 1 (1867) 479.

${ }^{80}$ OTR Berlin 13. 5. 1851, StriethorstA 2 (1856) $130=$ ZVersR 2 (1868) 302. Zu diesem Urteil vgl. bereits Hellwege $178 \mathrm{f}$. 
Der Versicherungsnehmer hatte diese Frist wegen ergebnisloser Vergleichsverhandlungen verstreichen lassen. Das Appellationsgericht Magdeburg war in der Vorinstanz noch von einer Verjährungsabrede ausgegangen und wandte daher ALR II $8 \$ 2355$ an. ${ }^{81}$ Das Obertribunal ordnete die Klausel als Bedingung ein. Dennoch verneinte es den Anspruch des Versicherungsnehmers nicht: Solange der Versicherer sich auf Vergleichsverhandlungen einlasse, habe der Versicherungsnehmer keinen Anlass zu klagen. Der Versicherer entbinde den Versicherungsnehmer vielmehr stillschweigend von der Erfüllung dieser Bedingung. Die Möglichkeit eines stillschweigenden Verzichts begegnete uns bereits im englischen Recht. ${ }^{82}$ Die Erklärung, die das Obertribunal für dieses Ergebnis anführte, war kurz und bezeichnend: ${ }^{83}$

»Die entgegengesetzte Annahme würde Treu und Glauben verletzen [...].«

Andere Verhaltensanforderungen an den Versicherungsnehmer wurden ebenfalls als Bedingungen im Sinne unseres heutigen $₫ 158$ BGB verstanden, wie ein Verfahren verdeutlicht, welches das Stadt- und Kreisgericht Danzig am 3.12. 1863 in erster Instanz und das Appellationsgericht Marienwerder am 7. 9.1864 in zweiter Instanz zu entscheiden hatte. Ein Gutspächter hatte sein Mobiliar am 20. 8. 1862 bei der Stettiner National-Versicherungsgesellschaft gegen Feuer versichern lassen. Am 28.10.1862 kam es zum Brand. Streitentscheidend war $\$ 6$ a der Versicherungsbedingungen, wonach »im Falle eines Brandes dem Versicherten die Verpflichtung auf[erlegt wird], binnen 14 Tagen nach dem Brande eine beglaubigte Abschrift der in Folge desselben aufgenommen polizeilichen Verhandlungen dem Agenten [...] einzusenden«. Nach $\ 13$ "zieht Nicht-Erfüllung oder auch nur unvollständige Erfüllung solcher Verpflichtungen den Verlust jedes Anspruches auf Entschädigung aus sämmtlichen mit der Gesellschaft geschlossenen Versicherungen nach sich«. Der Kläger übersandte die geforderte Abschrift nicht. Er glaubte, eine Erfüllung dieser Bestimmung sei entbehrlich, weil ein Vertreter der beklagten Versicherungsgesellschaft bei den Verhandlungen zugegen gewesen sei. Das Stadt- und Kreisgericht Danzig führte aus: ${ }^{84}$

"Die Einreichung der in Rede stehenden beglaubigten Abschriften hat nach $\$ .13$ a.a.O. die Bedeutung einer Bedingung, und zwar, da es sich wesentlich um eine Handlung des bedingt Berechtigten handelt, einer (positiven) Potestativ-Bedingung (\$. 100-105, Th. I, Tit. 4 des All. L.-R.). [...] Es ist aber ein feststehender Grundsatz, dass die Erfüllung, namentlich auch der Potestativ-Bedingung, schlechterdings erfolgen muss, ehe das bedingte Recht zur Existenz gelangen

81 Offenlassend dann AG Magdeburg 18. 5. 1867, ZVersR 2 (1868) 392.

82 Siehe oben N. 73.

${ }^{83}$ OTR Berlin 13.5. 1851 (oben N. 80), StriethorstA 2 (1856) $133=$ ZVersR 2 (1868) 305. Vgl. auch Stadtgericht Berlin 2. 3. 1866, PreußVersZ 1 (1867) 275.

${ }^{84}$ Stadt- und Kreisgericht Danzig 3. 12. 1863, Central-Organ 3 (1864) 65. 
kann ( $\$ .112$ u. 113, I, 4; $\$$. 491, I, 12 des Allg. L.-R.). Dies gilt selbst von nutzlosen Bedingungen (\$. 133, I, 4 a.a.O.).《

Daher sei es auch ohne Bedeutung, wenn der Zweck der Bedingung, wie vom Kläger behauptet, auf andere Weise erreicht werde. Das Appellationsgericht Marienwerder schloss sich der Argumentation des Kreis- und Stadtgerichts Danzig an. ${ }^{85}$ Zudem wurde es als bedeutungslos angesehen, wenn die Erfüllung dieser Verhaltensanforderung schuldlos unterblieb oder wenn die Erfüllung gar unmöglich war. ${ }^{86}$ Und noch 1867 sprach das Appellationsgericht Leipzig ganz in diesem Sinne mit Blick auf solche Klauseln von Resolutivbedingungen. ${ }^{87}$

Schließlich hatte sich die Rechtsprechung mit sogenannten Buchklauseln zu beschäftigen. Nach diesen war der Versicherungsnehmer gehalten, über seine versicherten, zumeist wechselnden Warenbestände Buch zu führen, und es war ihm nur gestattet, seinen Schadensnachweis durch die entsprechenden Geschäftsbücher zu führen. Noch 1865 ging das Stadt- und Kreisgericht Magdeburg davon aus, dass es sich bei einer solchen Klausel wiederum um eine Bedingung handelt. In der "Zeitschrift für Versicherungsrecht" ist die Urteilsbegründung wie folgt paraphrasiert: ${ }^{88}$

"Die Bedingung erschiene vielmehr als eine wesentliche, durch welche der Anspruch des Klägers selbst bedingt werde, so daß, da Kläger die Erfüllung der von ihm übernommenen Verpflichtung nicht nachzuweisen vermöge, er auch die Erfüllung der von der Beklagten nur bedingt übernommenen Verpflichtung nicht fordern könne."

Wir können mithin als Zwischenergebnis festhalten, dass die deutsche Rechtsprechung zunächst mit derjenigen in England übereinstimmte. Bis zur Mitte der 1860er Jahre wurden Verhaltensanforderungen, die an den Versicherungsnehmer nach Eintritt des Versicherungsfalls gestellt wurden, als Bedingungen eingeordnet. Nicht nur vereinzelte Gerichte folgten dieser Einordnung, sie bezog sich nicht nur auf ganz spezielle Verhaltensanforderungen und beruhte auch nicht auf partikularrechtlichen Besonderheiten, sondern war allgemein anerkannt und fand auch in der Literatur Unterstützung. ${ }^{89}$ Doch wandelte sich die Rechtsprechung zwischen 1865 und 1870 ebenso schlagartig wie grundlegend. ${ }^{90}$ Als Ergebnis bildete sich eine beson-

${ }^{85}$ AG Marienwerder 7. 9. 1864, Central-Organ n. F. 1 (1865) $296=$ ZVersR 1 (1866) 76. Ebenso OTR Berlin 25.3.1862, PreußVersZ 1 (1867) 681.

${ }^{86}$ OTR Berlin 25.3.1862 (vorige Note) 681.

87 AG Leipzig Juli 1867, Wochenblatt n.F. 16 (1868) 129 (134).

${ }^{88}$ Stadt- und Kreisgericht Magdeburg 28. 9. 1865, ZVersR 1 (1866) 151 (152).

${ }^{89}$ In diesem Sinne wohl Wilhelm Endemann, Das deutsche Handelsrecht (1865) 834.

${ }^{90}$ Anders Ralph Neugebauer, Versicherungsrecht vor dem Versicherungsvertragsgesetz (1990) 173 ff.; Tobias Prang, Der Schutz der Versicherungsnehmer bei der Auslegung von Versicherungsbedingungen durch das Reichsgericht (2003) 69ff., 80, $130 \mathrm{f}$., 190. Beide ordnen diese Änderung erst dem Reichsoberhandelsgericht und dem Reichsgericht zu. Dagegen setzt 
dere Art von Vertragsabreden heraus, die wir heute als Obliegenheiten bezeichnen.

\section{b) Entwicklungen ab 1865}

Am Anfang dieser Entwicklung stand eine schlichte Auflehnung gegen die Ergebnisse, welche die dogmatische Einordnung der Verhaltensanforderungen als Bedingungen eigentlich nach sich ziehen musste. So hatte sich das Stadtgericht in Frankfurt am Main im Jahre 1867 oder 1868 mit der Klausel auseinanderzusetzen, dass der Versicherungsnehmer einen Brand innerhalb von 24 Stunden anzuzeigen hatte: ${ }^{91}$ Durch den Frankfurter Dombrand in der Nacht vom 15. auf den 16. 8.1867 fing der Dachstuhl der Klägerin Sendelbach Feuer. Das bei der beklagten Dresdener Feuerversicherungsgesellschaft versicherte Mobiliar ging als Folge verloren. Die Beklagte lehnte eine Schadensregulierung ab. Die Klägerin hatte zwar vorgetragen, dass sie den Schaden am 16.8. und damit innerhalb von 24 Stunden angezeigt habe. Das wurde von der Beklagten indes bestritten. Die Anzeige sei erst am 17.8. erfolgt. Das Stadtgericht gab der Klage statt. Zwar betonte es, dass das Bestreiten der Beklagten, die Anzeige sei nicht am 16.8. erfolgt, verspätet vorgetragen worden sei, doch meinte es auch, dass eine Anzeige am 17. 8. noch genügt hätte. Es führte aus, dass

»[...] schonende Rücksicht zu nehmen [sei], als in Wirklichkeit das Bestreiten der klägerischen Ansprüche nur als eine Schikane und als ein aller Billigkeit Hohn sprechendes Pochen auf formale Stipulationen sich darstellt, während doch [...] feststeht, daß die Klägerin [...] den besten Willen zeigte, auch diesen streng formalen Verpflichtungen gerecht zu werden, der Beklagte dagegen sich nicht scheut, bei den an sich sehr strengen und schwer zu erfüllenden Bedingungen ein bis auf das Tüpfelchen vom I minutiöses Abwägen vorzunehmen, in Wahrheit also hier ein Fall vorliegt, wo der Richter der Natur der Sache nach und im Interesse der Billigkeit gehalten ist, einen den im Voraus festgestellten Bedingungen der Assecuranzgesellschaften, die das für den Abschluss von Versicherungsverträgen erlangte factische Monopol willkürlich auszubeuten sich in der Lage befinden, preisgegebenen Versicherungsnehmer nach Möglichkeit zu schützen «. ${ }^{92}$

Die Ausführungen drücken mehr die Empörung des Stadtgerichts aus, als dass sie eine dogmatische Begründung dafür geben, warum es nicht auf die

\footnotetext{
Rolf Peter Minnier, Das Alles-oder-nichts-Prinzip bei Obliegenheitsverletzungen im Privatversicherungsrecht (1967) 58 ff., diese Entwicklung früher an, ohne sie freilich zeitlich genau einzugrenzen. Nicht eindeutig Alexander Müssener, Die Entwicklung der Aachener Feuer-Versicherungs-Gesellschaft im 19. Jahrhundert unter besonderer Berücksichtigung ihrer Allgemeinen Geschäftsbedingungen (2007) $226 \mathrm{ff}$.

${ }^{91}$ Stadtgericht Frankfurt a. M. [ohne Datum], ZHR 12 (1868) 200. Zu diesem Urteil vgl. bereits Hellwege 172-175.

92 Stadtgericht Frankfurt a. M. [ohne Datum] (vorige Note) 200 (201f.).
} 
Erfüllung der Bedingung ankam: Es stellte auf den »streng formalen« Charakter der Verpflichtung und damit auf den Vertragsinhalt ab, es hielt das konkrete Vorgehen der Versicherung für schikanös und unbillig, und es stellte darauf $a b$, dass es sich um eine "im Voraus festgestellte Bedingung " handelte, welche die Versicherung unter Ausbeutung ihres faktischen Monopols dem Versicherungsnehmer aufdränge. Hier klingt die bereits spätere Monopolrechtsprechung des Reichsgerichts an. ${ }^{93}$ Dogmatisch ist die Begründung des Stadtgerichts nach alledem wenig greifbar. Zur gleichen Zeit beriefen sich Versicherungsnehmer vor anderen Gerichten darauf, dass entsprechende Klauseln ganz unwirksam seien, fanden hiermit freilich kein Gehör. $^{94}$

Vielmehr entwickelten Gerichte in ganz Deutschland eine andere Möglichkeit, unbillige Härten auszugleichen. Wir hatten oben gesehen, dass das Appellationsgericht Marienwerder und ebenso das Kreis- und Stadtgericht Danzig es noch im Jahr 1863 für unerheblich hielten, wenn der Versicherungsnehmer die Bedingung, den Schadensfall innerhalb einer bestimmten Frist anzuzeigen, deshalb nicht erfüllt hatte, weil der Versicherer von dem Schadensfall ohnehin Kenntnis hatte. ${ }^{95}$ Seit dem Jahr 1866 kristallisierte sich als vorherrschend die gegenteilige Ansicht heraus: ${ }^{96}$ Der Versicherer sollte sich dann nicht auf die Verletzung der genannten Abrede berufen können, wenn ihr Zweck anderweitig erreicht worden sei. Ein Urteil des Rheinischen Appellationsgerichtshofes zu Köln aus dem Jahr 1877 macht dies deutlich: ${ }^{97}$ Ein Christian Diener versicherte am 21.10. 1873 sein Haus mitsamt dem Mobiliar bei der North British and Merkantile Feuerversicherungs-Aktiengesellschaft. Die Police enthielt wiederum die Bestimmung, dass der Versicherte einen Brand innerhalb von 24 Stunden anzeigen, sich innerhalb von drei Tagen bei seiner Ortspolizeibehörde vernehmen lassen musste, das darüber aufgenommene Protokoll innerhalb von vierzehn Tagen bei dem Versicherer einreichen und innerhalb von vierzehn Tagen dem Versicherer eine Schadensaufstellung zukommen lassen musste. Am 17. 7. 1874

93 Hierzu ausführlich Hellwege 138-190.

94 So Kreisgericht Dessau 2.2.1866, ZVersR 2 (1868) 308. Vgl. Hellwege 153-155.

95 Siehe oben N.84.

96 Stadtgericht Frankfurt a.M. 29. 10. 1866, ZVersR 2 (1868) 207; AG Magdeburg 3. 11. 1866, ZVersR 2 (1868) 386; HAG Nürnberg 22. 11. 1866, zitiert von Conrad Malß, Uebersicht der neueren Rechtsprechung in nicht maritimen Versicherungssachen: ZHR 13 (1869) 418515 (440); OTR Berlin 4. 4. 1867, PreußVersZ 3 (1869) 988; AG Magdeburg 18. 5. 1867 (oben N. 81) 392; AG Insterburg 3.2. 1868, PreußVersZ 4 (1870) 243; AG Posen 8. 6. 1868, PreußVersZ 4 (1870) 5; OTR Berlin 5.11. 1868, PreußVersZ 4 (1870) 243; AGH Köln 21. 3. 1877, RheinA 68/1 (1877) 149; RG 15.6.1880, Gruchot 25 (1881) 1047; OLG Kiel 6.3. 1883, BuschA 46 (1886) 306. Vgl. auch schon OTR Berlin 24. 11. 1859, PreußVersZ 3 (1869) 847. Aus der Literatur Friedrich Meier, Ueber die Verbindlichkeiten des Versicherten bei und nach dem Brande: BuschA 30 (1874) 79-82 (80).

97 AGH Köln 21.3. 1877 (vorige Note) 149. 
brannte das versicherte Gebäude ab. Diener unterließ die Anzeige des Brandes und auch die Einreichung einer Schadensaufstellung. Doch war ein Agent des Versicherers bereits am Tag des Brandes am Ort des Geschehens gewesen, und auch von der Höhe des Schadens hatte der Versicherer auf andere Weise Kenntnis erlangt. Zudem hatte es Diener unterlassen, sich von der Ortspolizeibehörde vernehmen zu lassen und das Protokoll hierüber dem Versicherer zu übersenden. Indes hatte der Ortsbürgermeister Ermittlungen über die Brandursache aufgenommen und von sich aus die Ergebnisse an den Versicherer weitergeleitet. Eine Vernehmung des Versicherten sei, so das Gericht, daher entbehrlich gewesen, denn der Zweck der entsprechenden Policebedingung war erfüllt. In den Leitsätzen in der "Zeitschrift für Versicherungsrecht« zu einer Entscheidung des Stadtgerichts Frankfurt am Main aus dem Jahre 1866 heißt es plastisch, die »Wirkung der Clausel wird durch [ihren] Zweck begrenzt«. ${ }^{98}$ Zur Begründung verwies das Oberlandesgericht Kiel in einem Urteil aus dem Jahr 1883 auch auf die bona fides. ${ }^{99}$

Zudem bildete sich die Ansicht heraus, dass die an die Nichterfüllung einer Verhaltensanforderung geknüpfte Rechtsfolge nicht eingreift, wenn ihre Einhaltung dem Versicherungsnehmer unmöglich war, so etwa in einer Hamburger Rechtssache, die dem Oberappellationsgericht der freien Städte Deutschlands zu Lübeck 1867 zur Entscheidung vorlag: ${ }^{100}$ Die Firma Johannes Wacker \& Co. hatte ihr Lager bei der Deutschen Phönix und der Colonia versichert. Am 25. 5. $1857 \mathrm{kam}$ es zu einem Brand. Diesem fielen auch sämtliche Geschäftsbücher des Versicherungsnehmers zum Opfer. Strittig war die Schadenshöhe. Nach $\$ 11$ der Policebedingungen konnte der Versicherungsnehmer seinen Schaden nur durch Vorlage seiner Geschäftsbücher beweisen. Weil dies wegen des Brandes unmöglich sei, so das Oberappellationsgericht, könne eine Vorlage vom Versicherungsnehmer nicht verlangt werden. Er dürfe seinen Schaden daher anderweitig beweisen.

Und es setzte sich die Meinung durch, dass eine Nichterfüllung der Verhaltensanforderungen die an sie geknüpfte Rechtsfolge dann nicht auslöst, wenn die Nichterfüllung unverschuldet war. ${ }^{101}$ So ging es in einem Urteil des Bundesoberhandelsgerichts (BOHG) vom 4.4.1871 um die an einen Verstoß gegen $\$ 7$ der Allgemeinen Bedingungen der Feuer-Versicherungs-

98 Stadtgericht Frankfurt a. M. 29. 10. 1866 (oben N. 96) 207.

${ }^{99}$ OLG Kiel 6.3. 1883 (oben N. 96) 306.

100 OAG Lübeck 27. 6. 1867, Sammlung der Entscheidungen des Ober-Appellationsgerichts der freien Städte Deutschlands zu Lübeck 3 (1867) 550. Vgl. auch OLG Dessau 11.11. 1865, PreußVersZ 1 (1867) 385; OAG Dresden 4. 9. 1866, Annalen des Königlich Sächsischen Oberappellationsgerichts zu Dresden n.F. 3 (1868) 113; AG Insterburg 3.2.1868, 243; AG Posen 8. 6. 1868, 5 (beide oben N. 96); und das nicht ganz klare Urteil OAG Leipzig Oktober 1866, Wochenblatt n.F. 15 (1867) 5.

101 Vgl. etwa Niedergericht Hamburg 20.3. 1868, PreußVersZ 2 (1868) 703; OLG Kiel 25.1. 1883 (oben N. 96) 306; RG 8. 7. 1881, RheinA 72 (1882) 40; 4. 12.1903, RheinA 100/2 (1904) 100 . 
Gesellschaft Royal zu Liverpool geknüpften Rechtsfolgen. ${ }^{102}$ Dieser $\ 7$ enthielt wieder die üblichen Anzeigepflichten. $\$ 12$ sah sodann vor:

"Wenn der Versicherte - eine der dem Versicherten nach \$S 3, 4, 5, 6, 7, 9, 10 und 11 obliegenden Pflichten verletzt oder nicht vollständig erfüllt - so verliert der Versicherte jeden Anspruch auf Entschädigung."

Das Ostpreußische Tribunal zu Königsberg verstand in der Vorinstanz $₫ 7$ der Allgemeinen Bedingungen nicht mehr als Potestativbedingung. Denn nach $\$ 12$ derselben Bedingungen: ${ }^{103}$

"[...] ist (wie dies in Policen der meisten Gesellschaften zu sein pflegt) auf so viele Versäumnisse des Versicherten die Strafe des Verlustes seiner Ansprüche gesetzt, dass nothwendigerweise angenommen werden muss, dass alle diese Verletzungen eine Schuld des Versicherten voraussetzen. Es entspricht diese Annahme auch den gesetzlichen Bestimmungen über lex commissoria, Conventionalstrafe und Verzug, von deren Nachtheilen der Schuldner durch die anverschuldete Nichterfüllung oder Verspätung befreit wird. A.L.R.I. 16 \$S 17, 26. Ueberdies würde die Verklagte mit der entgegengesetzten Auslegung gegen $\$ 2024$ II. 8 A. L.R. handeln; es würde die Treue und Redlichkeit, zu welcher auch der Versicherer verpflichtet ist, verletzen, wenn sie die Police so verstehen wollte, dass es schließlich fast ganz ihrem Belieben überlassen bliebe, ob sie die Entschädigung zahlen will. Denn auch der sorgfältigste Versicherer wird kaum vermeiden können, in einen dieser Präjudizfälle des $₫ 12$ zu verfallen. Eine solche Auslegung würde übrigens auch dem $\$ 267$ I. 5 A.L.R., sowie, da hier ein Handelsgeschäft vorliegt, dem Art. 278 des Handelsgesetzbuchs widersprechen."

Als Folge könne sich der Versicherer nicht "auf die nackte Thatsache der nicht wörtlichen Innehaltung der Policebedingungen« berufen. ${ }^{104}$ Der zur Begründung unter anderem herangezogene ALR II 8 \$2024 lautet:

"Bey Schließung des Versicherungsvertrages sind beyde Theile zu besondrer Treue, Redlichkeit und Aufrichtigkeit verpflichtet; [...]."

Wir erinnern uns, dass Pollock C. B. in Mason v. Harvey auf Grundlage ganz ähnlicher Argumente, nämlich dass es dem Versicherer bei einer strikten Auslegung entsprechender Klauseln möglich sei, sich seiner Pflicht umfassend zu entziehen, kein Verschuldenserfordernis eingeführt hat, sondern lediglich solche Klauseln einschränkend auslegen wollte. ${ }^{105}$

Auch andere deutsche Gerichte begründeten ihre Entscheidungen unter Hinweis auf den guten Glauben, der bei Versicherungsverträgen in besonders hohem Maße zu berücksichtigen sei, so der Cassationshof Darmstadt im

102 BOHG 4. 4. 1871, Gruchot 15 (1871) 555.

103 Ostpreußisches Tribunal zu Königsberg [ohne Datum], Gruchot 15 (1871) 556.

104 Ostpreußisches Tribunal zu Königsberg [ohne Datum] (vorige Note) 556.

105 Siehe oben N. 65. 
Jahr 1865: ${ }^{106}$ Es ging wieder um die Anwendung einer Klausel, die dem Versicherungsnehmer auferlegte, innerhalb von vierzehn Tagen eine Schadensaufstellung einzureichen. Die Aufstellung des Versicherungsnehmers entsprach den Anforderungen nicht, der beklagte Versicherer verweigerte daher die Zahlung. Der Cassationshof machte sich die Ausführung des als Appellationsinstanz entscheidenden Obergerichts zu eigen: Würde man die üblichen Versicherungsbedingungen strikt auslegen, so müssten Versicherer kaum jemals zahlen, selbst wenn der Versicherungsnehmer ohne jede böse Absicht nicht im Einklang mit den Bedingungen handelte. Zweck der an den Versicherungsnehmer gestellten Verhaltensanforderungen sei nun aber allein, die Versicherer vor Betrug und Arglist auf Seiten des Versicherungsnehmers zu schützen, nicht aber, sie von jeder Haftung freizustellen. Dem guten Glauben entspreche allein, die Vertragsbedingung in diesem Sinne auszulegen. Und auch das Bundesoberhandelsgericht verwies 1871 auf ALR II 8 \$2024, der ein leitendes Prinzip des Versicherungsvertragsrechts normiere. ${ }^{107}$ Es setzte sich dabei bewusst über den Wortlaut der Versicherungsbedingungen hinweg, "als in der That eine rigoristische und lediglich den Wortlaut beachtende Interpretation der s.g. Policebedingungen dem Zwecke des Versicherungsinstituts, wie dem vernünftigen Willen redlicher $\mathrm{Pa}-$ ciscenten gleichmäßig widerstreiten würde $« .{ }^{108}$

\section{c) Zwischenergebnis}

Wir können nach alledem für das deutsche Recht zusammenfassen, dass es die Verhaltensanforderungen, die den Versicherungsnehmer nach Eintritt des Versicherungsfalles treffen, in Einklang mit dem englischen Recht zunächst als Bedingungen verstand mit der Folge, dass die für den Versicherungsnehmer nachteilige Rechtsfolge selbst dann eintrat, wenn dieser den Bedingungseintritt schuldlos nicht herbeigeführt hat und nicht herbeiführen konnte. Doch anders als in England vollzog sich in Deutschland zwischen den Jahren 1865 und 1870 in der Rechtsprechung schlagartig ein entscheidender Wandel, den auch die Literatur mit vollzog: ${ }^{109}$ Die in den Versicherungsbedingungen formulierten Bedingungen wurden teleologisch reduziert. Zudem sollten die an die Verletzung der Verhaltensanforderung

106 Cassationshof Darmstadt 26. 6. 1865, Sammlung der Entscheidungen des Großherzoglich Hessischen Cassationshofs in Civil- und Straf-Sachen 1865, 130. Ebenso RG 15. 6. 1880, 1047; Meier 80 (beides oben N. 96).

107 BOHG 4. 4. 1871 (oben N. 102) 557.

108 BOHG 4. 4. 1871 (oben N. 102) 558.

109 Wolff, Die Versicherungsbedingungen: ZVersR 2 (1868) 337-373 (363ff.); Otto Stobbe, Handbuch des deutschen Privatrechts ${ }^{2}$ III (1885) 362. Vgl. auch Kreis, Die Interessen der gegen Feuersgefahr Versicherten bei einer Neuordnung des Versicherungsprivatrechts: Gruchot 33 (1889) 481-501 (494ff.). Nicht eindeutig Heinrich Dernburg, Lehrbuch des Preußischen Privatrechts ${ }^{3}$ II (1882) 691. 
geknüpften Rechtsfolgen dann nicht eintreten, wenn es dem Versicherungsnehmer unmöglich war, sich mit ihr konform zu verhalten. Und schließlich wurde ein Verschuldenserfordernis in die Versicherungsbedingungen hineingelesen. Es war also die Rechtsprechung, welche die Grundlage für die im Versicherungsvertragsgesetz (VVG) von 1908 gefundene Lösung bereits in den 1860er Jahren legte, und nicht erst der Gesetzgeber des VVG, der das Verschuldensprinzip erstmalig zum Schutz der Versicherungsnehmer einführte. ${ }^{110}$

\section{Schlussbetrachtungen}

Diese Erkenntnisse werfen Folgefragen auf, denen hier nicht weiter nachgegangen werden kann:

1. So fällt auf, dass sich in Deutschland zu der uns interessierenden Frage erst später Fallrecht findet als in England, und es stellt sich die Frage nach dem Warum. Lagen die Gründe im Prozessrecht? ${ }^{111}$ Die Allgemeine Gerichtsordnung für die Preußischen Staaten (AGO) von 1793/1795 bestimmte in I $30 \$ 48$ :

"Wenn IV. aus einem Assekuranzkontrakte zwischen den Interessenten Streit entsteht, so soll die Sache, der Regel nach, in erster Instanz vor Schiedsrichtern verhandelt und entschieden werden. Doch ist den Parteien, oder auch einer derselben allein, unbenommen, auf die Entscheidung durch den ordentlichen Richter anzutragen."

Zudem waren die Rechtsmittel in Preußen eingeschränkt. In Versicherungssachen war eine Appellation möglich, eine Revision dagegen nach AGO I $30 \$ \$ 55-56$ ausgeschlossen, so dass nur die Nichtigkeitsbeschwerde blieb. Dort, wo die AGO nicht galt, war die dritte Instanz regelmäßig nach den Versicherungsbedingungen ausgeschlossen, so nach $\$ 61$ der Statuten der Gothaer Feuerversicherungsbank für Deutschland: ${ }^{112}$

"In allen Fällen, in welchen Entscheidung der Gerichtsbehörden eintritt, ist gegen das Erkenntnis der ersten Instanz nur das ordentliche Rechtsmittel der Ap-

110 So aber etwa Münch. Komm. VVG (-Wandt) \28 Rz. 5. Allgemein schreibt auch $A n$ gela Duvinage, Die Vorgeschichte und die Entstehung des Gesetzes über den Versicherungsvertrag (1987) 201 ff., dem VVG-Gesetzgeber eine besondere Rolle bei der Einschränkung der Vertragsfreiheit auf dem Gebiet des Versicherungsvertragsrechts zu. Vgl. weiterhin Motive zum Versicherungsvertragsgesetz, hrsg. vom Bundsaufsichtsamt für das Versicherungs- und Bausparwesen (Neudruck 1967) $80 \mathrm{ff}$.

${ }^{111}$ Vgl. OTR Berlin 27.11.1851, Entscheidungen des königlichen Ober-Tribunals 2/2. Folge (1852) 283; Carl Friedrich Eichhorn, Einleitung in das deutsche Privatrecht ${ }^{5}$ (1845) 320; Johann Caspar Bluntschli, Deutsches Privatrecht ${ }^{3}$ (1864) 501.

112 Wiedergegeben in ZVersR 2 (1868) 177. 
pellation oder Berufung an die nächste Instanz zulässig, bei deren Entscheidung es sein Bewenden behält.«

Gerichte erkannten solche Klauseln an. ${ }^{113}$ Es mag also sein, dass sich die deutschen Gerichte ebenso früh mit Verhaltensanforderungen im Feuerversicherungswesen auseinandersetzten wie die englischen Gerichte, dass die entsprechenden unter- und schiedsgerichtlichen Urteile nur nicht veröffentlicht wurden. Freilich passt zu diesem Erklärungsversuch nicht, dass sich auch in den Versicherungsbedingungen englischer Versicherer vergleichbare Klauseln fanden. ${ }^{114}$ Wahrscheinlicher ist, dass das zeitlich verzögerte Einsetzen der Rechtsprechung in Deutschland schlicht die Entwicklung des privaten Feuerversicherungswesens widerspiegelt. ${ }^{115}$

2. Zudem ist augenfällig, dass in der deutschen und englischen Versicherungspraxis vergleichbare, ja zum Teil identische Klauseln auftraten. Dies könnte darin seine Erklärung finden, dass englische Versicherer auch in Deutschland tätig waren, wie nicht zuletzt Fälle vor deutschen Gerichten mit Beteiligung englischer Versicherer belegen. ${ }^{116}$ Englische Versicherer könnten ihre in der Heimat bewährten Bedingungen mitgebracht und so die deutsche Feuerversicherungspraxis beeinflusst haben.

3. Dass englische Feuerversicherer auch in Deutschland aktiv waren, legt die Vermutung nahe, dass ein, modern gesprochen, europäischer Binnenmarkt für Versicherungen im 19. Jahrhundert im Ansatz funktioniert hat. Es bestanden sicherlich Hürden, so die Konzessionspflicht für den Betrieb einer Versicherung in vielen deutschen Staaten. ${ }^{117}$ Aber das Versicherungsvertragsrecht stellte eine solche Hürde offenbar zunächst nicht dar, konnten wir doch zumindest für Verhaltensanforderungen, die an den Versicherungsnehmer nach Eintritt des Schadensfalls gestellt werden, bis zur Mitte des 19. Jahrhunderts eine Übereinstimmung zwischen deutschem und englischem Recht feststellen.

4. Das wirft die Frage auf, ob diese Übereinstimmung Zufall war: ${ }^{118}$ Nicht nur die Versicherungspraxis machte bis zur Mitte des 19. Jahrhunderts nicht an den Grenzen einzelner Rechtsordnungen halt. Auch die versicherungsvertragsrechtliche Gesetzgebung der einzelnen Staaten fügte sich bis

113 Vgl. OAG Dresden 8. 8. 1850, ZVersR 2 (1868) 176, mit Hinweisen auf OTR Berlin 1.2. 1845 und OAG Dresden 16.10. 1850.

${ }^{114}$ Vgl. Beaumont (oben N. 57) 91; Bunyon, Fire Insurance 3 159; Ziffer X der Versicherungsbedingungen der Protector Fire Insurance Co., abgedruckt bei Ellis, Law of Fire (oben N. 55) 228.

115 Vgl. nur Neugebauer (oben N. 90) 13 ff.; Louis Pahlow, Ein "Geschöpf der ganzen civilisierten handeltreibenden Welt«: Zeitschrift für Neuere Rechtsgeschichte (ZNR) 29 (2007) $18-46$.

116 Vgl. BOHG 4. 4. 1871 (oben N. 102) 555; AGH Köln 5. 7.1876, RheinA 68/1 (1877)

53; 21. 3. 1877 (oben N. 96) 149. Vgl. außerdem Müssener (oben N. 90 ) $32 \mathrm{f}$.

117 Vgl. nur Hellwege 167 ff. m. w. N.

118 Zum Folgenden nur Schneider (oben N. 19) 41 ff.; Hellwege $62 \mathrm{f}$. 
in das 19. Jahrhundert hinein in einen gemeineuropäischen Rahmen ein. Die versicherungsrechtliche Literatur wurde ebenfalls in ganz Europa rezipiert. Und schließlich wurde die europäische Rechtsprechung zumindest von deutschen Gerichten als Erkenntnisquelle beachtet, wie ein Beispiel aus dem Lebensversicherungsrecht zeigt. Die Deutsche Lebens-VersicherungsGesellschaft berief sich vor dem Oberappellationsgericht der vier freien Städte Deutschlands zu Lübeck darauf, dass eine bestimmte Frage des Lebensversicherungsrechts im Sinne der englischen Gerichtspraxis zu beantworten sei. Das Gericht führte aus: ${ }^{119}$

"Das was im Auslande, und insonderheit in England in Betreff der Lebens-Versicherungen geltendes Recht ist, kann mithin nur aushülfsweise und allein insofern in Betracht kommen, als darin Regeln enthalten sind, welche in einem verwandten Rechtsgebiete durch Anerkennung Seitens der Betheiligung und der Gerichte, sowie durch wissenschaftliche Begründung Seitens der Rechtslehrer, in gewissem Grade eine Autorität erlangt haben."

Das Oberappellationsgericht wollte sich der englischen Rechtsprechung nicht unbesehen anschließen. Aber der Fall offenbart, dass sich zumindest Versicherer auf englisches Fallrecht beriefen und dass deutsche Gerichte dieses noch zur Mitte des 19. Jahrhunderts nicht als vollkommen unbeachtlich beiseiteschoben. Und so ist es möglich, dass die identische dogmatische Einordnung von Verhaltensanforderungen, die nach Eintritt des Versicherungsfalles an den Versicherungsnehmer gestellt wurden, Folge eines gegenseitigen oder auch nur einseitigen Austausches zwischen englischem und deutschem Recht war.

5. Aber wie kam es zu dem abrupten Wandel im deutschen Recht? Und warum vollzog sich dieser Wandel nicht auch in England? Die Antworten auf diese Fragen sind wohl wieder in der Entwicklung des Feuerversicherungswesens zu suchen: In England gab es privat betriebene Feuerversicherungen früher als in Deutschland. Entsprechend kristallisierte sich frühzeitig eine ständige Rechtsprechung zur dogmatischen Einordnung der an den Versicherungsnehmer gestellten Verhaltensanforderungen heraus. Dies geschah zu einer Zeit, als die Angst vor einem Versicherungsbetrug durch den Versicherungsnehmer im Vordergrund stand und das Bewusstsein, dass der Versicherungsnehmer Schutz vor der Praxis der Versicherer bedarf, noch unterentwickelt war. Das englische Fallrechtssystem machte eine nachfolgende Rechtsprechungsänderung nur schwer möglich.

In Deutschland treten uns die ersten Fälle zu der uns interessierenden Frage überhaupt erst zur Mitte des 19. Jahrhunderts entgegen. Aber schon zu Beginn der 1860er Jahre wurde betont, dass auch der Versicherungsnehmer schutzwürdig sei, wie etwa die Diskussionen um die versicherungsrecht-

119 OAG Lübeck 30. 11. 1857, Die Jurisprudenz des Oberappellationsgerichts der vier freien Städte Deutschlands in bürgerlichen Rechtssachen aus Lübeck 2 (1866) 390 (391). 
lichen Vorschriften des Dresdener Entwurfs von 1866 eindrucksvoll belegen. ${ }^{120}$ Dieser Perspektivenwechsel ereignet sich in Deutschland also, noch bevor sich die Rechtsprechung gefestigt hatte.

Freilich konnten wir sehen, dass man auch in England seit der zweiten Hälfte des 19. Jahrhunderts ein Bedürfnis danach erkannte, Versicherungsnehmer zu schützen. Und auch bei den Schutzmechanismen konnten wir Übereinstimmungen feststellen. So trat uns der Gedanke, dass sich der Versicherer nicht auf die Nichteinhaltung einer Verhaltensanforderung berufen kann, wenn er auf ihre Erfüllung verzichtet hat, in beiden Rechten entgegen. Nur vollzog die deutsche Rechtsprechung nachfolgend einen grundlegenden Wandel, während die englische Rechtsprechung weiterhin versuchen musste, einen Schutz des Versicherungsnehmers mit einer Vielzahl unterschiedlicher Instrumente, etwa dem stillschweigenden Verzicht oder einer einschränkenden Auslegung, zu gewährleisten, ohne das Verständnis der Verhaltensanforderungen als condition precedent in Frage zu stellen. Freilich können diese Mechanismen den Schutz des Versicherungsnehmers nur unvollkommen gewährleisten, steht doch etwa bei der Frage, ob der Versicherer auf die Einhaltung der Verhaltensanforderungen stillschweigend verzichtet hat, allein dessen Verhalten im Blick und nicht die Schutzbedürftigkeit des Versicherungsnehmers.

6. Können die gewonnenen Erkenntnisse einen Dialog zwischen englischem und deutschem Recht erleichtern und den Weg hin zu einem europäischen Versicherungsvertragsrecht ebnen? Das deutsche und englische Recht hatten einen identischen Ausgangspunkt. Beiden Rechten war das Verschuldenserfordernis zunächst fremd. In Deutschland wurde es von der Rechtsprechung entwickelt und unter anderem daran festgemacht, dass Versicherungsverträge in besonderem Maße Treu und Glauben unterstehen. Aber auch im englischen Recht werden Versicherungsverträge als Verträge of utmost good faith verstanden. Obwohl sich daraus für beide Parteien besondere Treuepflichten ergeben sollen, ${ }^{121}$ hat die englische Rechtsprechung bisher einseitig diejenigen des Versicherungsnehmers betont: ${ }^{122}$

"Nonetheless, the tendency of the courts and the customary practice in the insurance market on this issue have been far from the principle of mutuality. In other words, this duty has rarely been applied to an insurer."

Der Verweis auf die besonderen Treuepflichten auch des Versicherers könnte der Schlüssel sein, englische Juristen von dem Verschuldenserforder-

${ }^{120}$ Vgl. hierzu umfassend Kübel, Die Verhandlungen der Dresdener Bundeskommission zur Ausarbeitung eines allgemeinen deutschen Obligationsrechts über den Versicherungsvertrag: ZVersR 2 (1868) 1-104.

${ }_{121}$ Drake Insurance plc. v. Provident Insurance plc. (oben N. 28) 1834.

${ }^{122}$ Park (oben N. 19) para. 2.2.3.1.2. Vgl. MacDonald Eggers/Picken/Foss paras. 3.80ff.; Birds para. 7.0. 
nis zu überzeugen. Zwar werden englische Juristen betonen, dass dies dem Wortlaut der Versicherungsbedingungen zuwiderlaufe. Doch deutete sich bereits im 19. Jahrhundert an, dass englische ebenso wie deutsche Gerichte bereit sind, sich mit Blick auf die besonderen Treuepflichten der Parteien über den Wortlaut des Vertrages hinwegzusetzen. ${ }^{123}$

7. Schließlich können wir festhalten, dass die historische Rechtsvergleichung auch im Versicherungsvertragsrecht gewinnbringend eingesetzt werden kann. Sie steht hier freilich auf einem anderen Fundament. Zwar spielt das römisch-kanonische ius commune als gemeinsame Wurzel der europäischen Versicherungsvertragsrechte eine nur marginale Rolle. Gemeinsame Wurzeln existieren dennoch. Sie finden ihren Grund in der Entwicklung des Versicherungswesens und der Versicherungspraxis. Sie gilt es herauszuarbeiten. Freilich kommt es wegen des unterschiedlichen Fundaments auch zu einem Perspektivenwechsel: Ist es für das allgemeine Zivilrecht zulässig, die Schriften der europaweit rezipierten Lehrbücher des ius commune in das Zentrum der Forschung zu stellen, so geht es im Versicherungsvertragsrecht auch darum, die gemeinsame Praxis der Versicherer, die in den Versicherungsbedingungen hervortritt, und die Linien der Rechtsprechung in den einzelnen europäischen Rechtsordnungen herauszuarbeiten.

\section{Summary}

\section{Condition Precedent in Insurance Contract Law in Historical and Comparative Perspective}

Both English and German law first qualified those terms which oblige an assured after the occurrence of the loss, such as giving notice of the loss within a certain time, as conditions precedent. English courts still uphold this qualification. As a consequence, the insurer may refuse payment under the policy even if the assured has, without being at fault, not fulfilled such condition precedent. In contrast, German case law saw a dramatic change between 1865 and 1870. Since 1865, German courts have developed the view that it is sufficient if the purpose of the term has been fulfilled, even if there is not strict compliance with the term. Furthermore, if fulfilment of the term was no longer possible, then this would be taken into consideration by the courts. Finally, it was held that the insurer may refuse payment under the policy only if the assured was at fault. By way of justification, the German courts referred to the nature of the contract as one of utmost good faith.

The observation that both English and German law share a common root is important. Firstly, it proves that the method of comparative legal history may also be fruitfully applied to insurance law, an approach that has been

${ }^{123}$ Siehe oben den Text zu und nach N. 68. 
forcefully promoted by Reinhard Zimmermann. However, within insurance law the focus of comparative-historical research needs to be on the development of insurance practice and the case law. Secondly, in the context of insurance contract law the findings of comparative and historical research may also be useful to bridge the gap between the continental civil law and the English common law. 